Dicle University Journal of Engineering (DUJE)

Araştırma Makalesi / Research Article

\title{
Gauss ve Laplace Gürültülü Kanallar Arasında Optimal Anahtarlama
}

\section{Optimal Channel Switching between Gauss and Laplace Channels}

\author{
M. Emin Tutay ${ }^{1}$ \\ ${ }^{1}$ Dicle Üniversitesi, Elektrik-Elektronik Mühendisliği Bölümü, Diyarbakır, emin.tutay@dicle.edu.tr ORCID:0000-0002-1506-0028
}

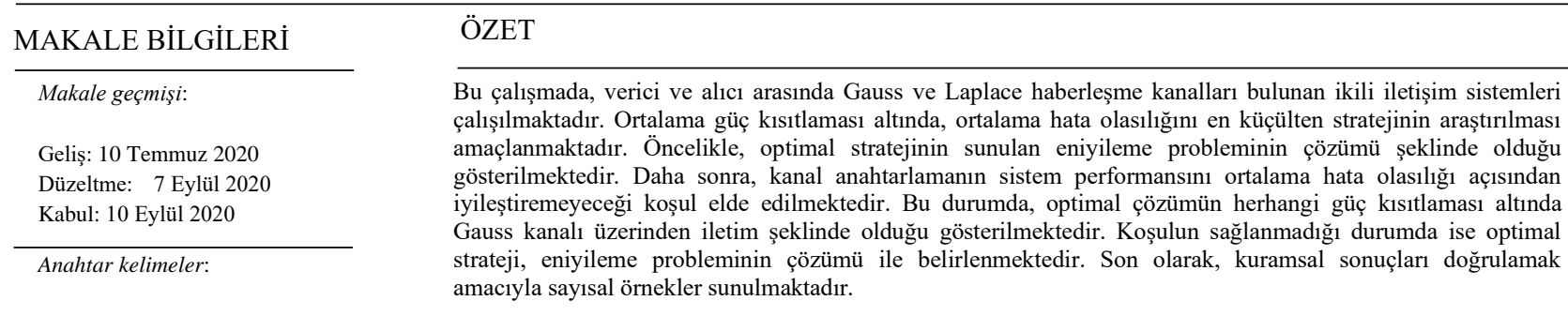

Kanal anahtarlama, ortalama hata olasılı̆̆ı, Gauss kanalı, Laplace kanalı

\begin{tabular}{|c|c|}
\hline \multicolumn{2}{|c|}{ ARTICLE INFO } \\
\hline \multicolumn{2}{|c|}{ Articlehistory: } \\
\hline Received: & 10 July 2020 \\
\hline Revised: & 7 September 2020 \\
\hline Accepted: & 10 September 2020 \\
\hline \multicolumn{2}{|l|}{ Keywords: } \\
\hline $\begin{array}{l}\text { Channel s } \\
\text { error pr } \\
\text { channel, } \mathrm{La}\end{array}$ & $\begin{array}{l}\text { witching, average } \\
\text { obability, Gauss } \\
\text { place channel }\end{array}$ \\
\hline
\end{tabular}

\begin{abstract}
In this paper, binary communication systems are studied in the presence of Gauss and Laplace channels. The objective is to investigate the optimal strategy that minimizes average error probability under the average power constraint. First, it is shown that the optimal strategy is the solution of the presented optimization problem. Then, the condition in which channel switching does not provide any further performance enhancement is obtained. In this case, it is shown that the optimal strategy is to transmit over Gauss channel for any value of the average power constraint. In the case of the condition is not satisfied, the optimal strategy is determined by the solution of the optimization problem. Finally, to corroborate theoretical results, numerical examples are presented.
\end{abstract}




\section{Giriş}

Illetişim sistemlerinde, ortalama güç kısıtlaması altında rastgeleleştirme tekniğinin, performansı ortalama hata olasılığı açısından iyileştirebildiği literatürde çeşitli çalışmalarda gösterilmiştir [110]. Gauss olmayan gürültü dağılımları için bu yaklaşım, sitemin verici tarafında düşünülmüş ve optimal işaretleme stratejisi belirlenmiştir [1-3]. Aynı yaklaşım, iletişim sistemlerinin alıcı tarafında da ele alınmış ve optimal sezici rastgeleleştirme tekniği ile beraber bunlara karşılık gelen optimal işaretleme stratejileri çalışılmıştır [6-7].

Sistemde verici ve alıcı arasında birden çok haberleşme kanalı bulunması durumunda, rastgeleleştirme tekniği farklı haberleşme kanallarının iletişim için zaman paylaşımlı kullanılması şeklinde uygulanabilir [8-10]. Literatürde kanal anahtarlama olarak bilinen bu teknik, ilk olarak [8]'de ele alınmış, sistemin ortalama hata olasılığının kanal gürültü modellerine ve ortalama güç kısıtlamasına bağlı olarak iyileştirilebileceği gösterilmiştir. Bir başka çalışmada, Gauss gürültülü kanal ile Gauss karışım gürültülü kanallar ele alınmış ve ortalama hata olasılığının iyileştirilebildiği çeşitli örnekler ile gösterilmiştir [9].

$\mathrm{Bu}$ çalışmada, sistemdeki toplanır gürültülü kanal modellerinin Gauss veya Laplace gürültülü kanal modelleri olduğu ikili iletişim sistemi, güç kısıtlaması altında ele alınmaktadır. İlk olarak, optimal stratejinin sunulan eniyileme probleminin formülü şeklinde olduğu gösterilmektedir. Daha sonra, Gauss gürültülü kanal ile Laplace gürültülü kanala göre asimptotik olarak daha iyi performans elde edildiği gösterilmektedir. Ayrıca, Gauss ve Laplace kanalları arasında rastgeleleştirmenin tek kanallı iletime göre ortalama hata olasılığını iyileştiremediği koşul elde edilmektedir. Bu durumda, ortalama güç kısıtlamasının değerinden bağımsız olarak optimal stratejinin Gauss kanalı üzerinden iletim şeklinde olduğu gösterilmektedir. Belirtilen koşulun sağlanmadığı durumda, sistemin güç kısıtlamasına bağlı olarak Gauss veya Laplace gürültülü kanalların birbirlerine göre daha iyi performans verebilecekleri gösterilmektedir. Bu durumda, optimal strateji eniyileme probleminin çözümü ile elde edilmektedir. Son olarak, rastgeleleştirmenin ortalama hata olasılığını iyileştirdiği ve iyileştirmediği her iki durum için sayısal sonuçlar birer örnek ile gösterilmektedir.

\section{Problem Tanımı}

İletişim sistemlerinde, verici ve alıcı arasında anlık olarak sadece birinin kullanıldığı çoklu toplanır gürültü kanallarının mevcut olduğu durum ele alınmaktadır. Belirli bir anda, verici hangi haberleşme kanalının kullanılacağı bilgisine sahip olup, bu kanala karşılık gelen yıldız kümesine (signal constellation) göre iletimi gerçekleştirmektedir.

Kanal gürültülerinin, Gauss veya Laplace dağglımına sahip gürültüler olduğu ikili iletişim sistemleri düşünülmektedir. Gürültü dağılımları Gauss ve Laplace ile modellenen kanal sayıları, sırasıyla $K_{G}$ ve $K_{L}$ ile gösterilmektedir. Burada, $K_{G}$ adet Gauss kanalı değişinti (varyans) değerleri açısından farklılık göstermektedir. Benzer bir şekilde $K_{L}$ adet Laplace kanalı da değişinti değerleri açısından farklılık göstermektedir. Ayrıca, "0" ve "1" sembollerine karşılık gelen önsel olasılıkların eşit olduğu kabul edilmektedir. $\mathrm{Bu}$ durumda, en iyi performansı verdiğinden alıcıda maksimum olabilirlik (ML) kurallı sezici kullanılmaktadır [11-12].

$\mathrm{Bu}$ çalışmada amaç, ortalama güç kısıtlaması altında ortalama hata olasılığını minimize eden stratejiyi bulmaktır. Mevcut kanal sayısından bağımsız olarak, optimal stratejinin en çok iki kanal üzerinden zaman paylaşımı şeklinde olduğu $[4,8,10]$ çalışmalarındakine benzer yaklaşımlarla ispat edilebilir. Ayrıca, ortalama hata olasılığı Gauss ve Laplace kanalları için tekdüze, azalan ve dişbükey fonksiyonlar olduğundan optimal strateji için en düşük değişintili Gauss ve Laplace kanalları dışındakilerin kullanılmayacağı açıktır.

Matematiksel olarak ifade edilecek olursa, $U=\left\{\alpha, P_{G}, P_{L}\right\}$ eniyileme uzayı iken, aşağıda belirtilen eniyileme probleminin çözümü optimal stratejiyi belirleyecektir. 


$$
\begin{aligned}
& \min _{U} \alpha e_{G}\left(P_{G}\right)+(1-\alpha) e_{L}\left(P_{L}\right) \\
& \text { öyle ki } \quad \alpha P_{G}+(1-\alpha) P_{L} \leq P_{a v g} \\
& 0 \leq \alpha \leq 1
\end{aligned}
$$

Burada $P_{G}$ ve $P_{L}$ sirasiyla Gauss ve Laplace kanalları üzerinden yapılan iletim için harcanan ortalama gücü gösterirken $e_{G}\left(P_{G}\right)$ ve $e_{L}\left(P_{L}\right)$ buna karşıllk elde edilen ortalama hata olasılıklarını göstermektedir. Sistem için ortalama güç kısitlaması $P_{a v g}$ ile belirtilmektedir.

Ayrıca, $\alpha$ iletim için Gauss kanalının kullanılma oranını gösteren değişken (kanal anahtarlama oranı) olarak, tanımlanmaktadır. Örnek olarak, $\quad \alpha=0.2$ değeri zamanın $\% 20$ 'sinde Gauss kanalı üzerinden iletim yapıldığ $1, \% 80$ 'inde ise Laplace kanalı üzerinden iletim yapıldığı anlamını taşımaktadır.

Mevcut gürültü dağılımları ve eşit önsel olasılıklar dikkate alındığında, "0" ve "1" bitleri için $S_{0}$ ve $S_{1}$ işaret değerlerini göstermek üzere, işaret değerleri $S_{1}=-S_{0}$ olarak seçileceğinden, denklem (1)'deki ortalama hata olasılıkları iletim için harcanan ortalama gücün fonksiyonu olarak belirtilmektedir.

Denklem (1) incelendiğinde, optimal stratejinin aşağıda belirtilen yöntemlerden biri şeklinde olduğu görülmektedir:

- Gauss kanalı üzerinden iletim $(\alpha=1)$

- Laplace kanalı üzerinden iletim $(\alpha=0)$

- Gauss ve Laplace kanalları üzerinden zaman paylaşımı şeklinde iletim $(0<\alpha<1)$

\section{Optimal Strateji}

$\mathrm{Bu}$ bölümde optimal strateji için yukarıda belirtilen yöntemler incelenmektedir. Ortalama değeri "0" olan Gauss gürültüsü için olasılık yoğunluk fonksiyonu (OYF)

$$
f_{G}(n)=\frac{1}{\sqrt{2 \pi} \sigma_{G}} e^{-\frac{n^{2}}{2 \sigma_{G}^{2}}}
$$

olarak tanımlanmaktadır [13]. Burada $\sigma_{G}$ Gauss gürültüsü için standart sapma değerini göstermektedir. Bu kanal üzerinden $P_{G}$ ortalama gücü harcanarak yapılan ikili iletişim neticesinde ortalama hata olasılığ $e_{G}\left(P_{G}\right)=Q\left(\sqrt{P_{G}} / \sigma_{G}\right)$ olarak hesaplanmaktadir [13]. Burada $Q(x)=\frac{1}{\sqrt{2 \pi}} \int_{x}^{\infty} e^{-t^{2} / 2} d t$ şeklinde tanımlanmaktadır [13].

Ortalama değeri “0” olan Laplace gürültüsü için OYF,

$$
f_{L}(n)=\frac{1}{\sqrt{2} \sigma_{L}} e^{-\frac{|n| \sqrt{2}}{\sigma_{L}}}
$$

olarak verilmektedir [13]. Burada $\sigma_{L}$ Laplace gürültüsü için standart sapmayı göstermektedir. Bu kanal üzerinden $P_{L}$ ortalama gücü harcanarak elde edilen ortalama hata olasilığ $\frac{1}{2} e^{-\sqrt{2 P_{L}} / \sigma_{L}}$ olarak hesaplanabilir.

Optimal stratejinin belirlenebilmesi amaciyla, iki kanalın ortalama hata olasılıkları arasındaki fark aşağıdaki fonksiyon olarak tanımlanmaktadır:

$$
\begin{aligned}
e_{d}(p) & =e_{L}(p)-e_{G}(p) \\
& =\frac{1}{2} e^{\frac{-\sqrt{2 p}}{\sigma_{L}}}-Q\left(\frac{\sqrt{p}}{\sigma_{G}}\right)
\end{aligned}
$$

Türev alınacak olursa,

$$
e_{d}^{\prime}(p)=\frac{1}{2 \sqrt{2 p}}\left[\frac{e^{-p / 2 \sigma_{G}^{2}}}{\sigma_{G} \sqrt{\pi}}-\frac{e^{-\sqrt{2 p} / \sigma_{L}}}{\sigma_{L}}\right]
$$

eşitliği elde edilmektedir.

Lemma 1: Gauss ve Laplace kanallarının ortalama hata olasılıkları için, aşağıda verilen eşitsizlik geçerlidir:

$$
e_{G}(p)<e_{L}(p) \forall p \in\left(\frac{8 \sigma_{G}^{4}}{\sigma_{L}^{2}}, \infty\right)
$$

İspat: Kanalların ortalama hata olasılık performansları için aşağıdaki eşitsizlikler geçerlidir:

$$
e_{G}(p) \stackrel{(1)}{\leq} \frac{1}{2} e^{\frac{-p}{2 \sigma_{G}^{2}}}<\frac{12}{2} e^{\frac{-\sqrt{2 p}}{\sigma_{L}}}=e_{L}(p)
$$

Burada, eşitsizlik (1), $Q($.$) fonksiyonu için$ geçerli olan $Q(x) \leq 0.5 e^{\frac{-x^{2}}{2}}$ eşitsizliğinden dolayı 
geçerlidir [14]. Eşitsizlik (2) ise önermede belirtilen $p>8 \sigma_{G}^{4} / \sigma_{L}^{2}$ koşuluna dayanmaktadır.

Lemma 1, güç seviyesinin belirli bir sınırın üstünde olması durumunda Gauss kanalı ile Laplace kanalına göre daima daha düşük ortalama hata olasılığının elde edildiğini ifade etmektedir. Belirtilen neticenin geçerli olması, güç için her iki kanalın değişintileri ile belirlenen bir alt sınırı gerektirmektedir.

Önerme 1: Gauss ve Laplace kanal gürültülerinin standart sapma değerleri $\sigma_{L} \geq \sigma_{G} \sqrt{\pi}$ eşitsizliğini sağlıyorsa, denklem (1)'de tanımlanan problem için optimal strateji Gauss kanalı üzerinden iletim yapmaktır.

İspat: $e_{d}^{\prime}(p)$ fonksiyonu incelendiğinde,

$P^{*}=\frac{2 \sigma_{G}^{4}}{\sigma_{L}^{2}}+2 \sigma_{G}^{2}\left[\frac{\sigma_{G}^{2}}{\sigma_{L}^{2}}-\ln \left(\frac{\sigma_{G} \sqrt{\pi}}{\sigma_{L}}\right)\right]+4 \frac{\sigma_{G}^{3}}{\sigma_{L}}\left[\frac{\sigma_{G}^{2}}{\sigma_{L}^{2}}-\ln \left(\frac{\sigma_{G} \sqrt{\pi}}{\sigma_{L}}\right)\right]^{1 / 2}$

olmak üzere, fonksiyonun $\left[0, P^{*}\right)$ aralığında pozitif, $\left(P^{*}, \infty\right)$ aralığında ise negatif değere sahip olduğu gösterilebilir [15]. Ayrıca, Lemma 1 'in sonucu dikkate alındiğında, $e_{d}(p)>0 \quad \forall p>0$ sonucu elde edilmektedir. Dolayısıyla, eşit güç harcandığında Gauss kanalı daima daha iyi performans göstermektedir.

Önerme 1, kanal gürültüleri için $\sigma_{L} \geq \sigma_{G} \sqrt{\pi}$ şartı sağlandığında denklem (1)'de belirtilen eniyileme problemi için optimal çözümün Gauss kanalı üzerinden iletim şeklinde olduğunu ifade etmektedir.

Önerme 2: Kanal gürültülerinin standart sapma değerleri için $\sigma_{L}<\sigma_{G} \sqrt{\pi}$ eşitsizliği geçerli olduğunda, $e_{d}(p)=0$ eşitliğini sağlayan sonlu tek bir pozitif değer $\left(p^{\dagger}\right)$ olup kanal performansları arasındaki ilişki aşağıda belirtilmektedir:

$$
\begin{array}{ll}
\text { - } & e_{L}(p)<e_{G}(p), p<p^{\dagger} \\
\text { - } & e_{L}(p)>e_{G}(p), p>p^{\dagger}
\end{array}
$$

İspat: $e_{d}^{\prime}(p)$ fonksiyonu incelendiğinde $p_{1}$ ve $p_{2}$, $p_{1}=\frac{2 \sigma_{G}^{4}}{\sigma_{L}^{2}}+2 \sigma_{G}^{2}\left[\frac{\sigma_{G}^{2}}{\sigma_{L}^{2}}-\ln \left(\frac{\sigma_{G} \sqrt{\pi}}{\sigma_{L}}\right)\right]-4 \frac{\sigma_{G}^{3}}{\sigma_{L}}\left[\frac{\sigma_{G}^{2}}{\sigma_{L}^{2}}-\ln \left(\frac{\sigma_{G} \sqrt{\pi}}{\sigma_{L}}\right)\right]^{1 / 2}(10 a)$ $p_{2}=\frac{2 \sigma_{G}^{4}}{\sigma_{L}^{2}}+2 \sigma_{G}^{2}\left[\frac{\sigma_{G}^{2}}{\sigma_{L}^{2}}-\ln \left(\frac{\sigma_{G} \sqrt{\pi}}{\sigma_{L}}\right)\right]+4 \frac{\sigma_{G}^{3}}{\sigma_{L}}\left[\frac{\sigma_{G}^{2}}{\sigma_{L}^{2}}-\ln \left(\frac{\sigma_{G} \sqrt{\pi}}{\sigma_{L}}\right)\right]^{1 / 2}(10 b)$

yukarıda belirtilen değişkenler olmak üzere, fonksiyonun $\left(p_{1}, p_{2}\right)$ aralığında pozitif değer aldığı, bunun dişında ise $\left(\left[0, p_{1}\right) \cup\left(p_{2}, \infty\right)\right)$ negatif değer aldığı gösterilebilir [15]. $e_{d}(p)$, $p=0$ için " 0 ” değerini aldığından, $e_{d}(p)$ fonksiyonunu " 0 " yapan sonlu tek bir değer olup, $\quad p^{\dagger} \in\left(p_{1}, p_{2}\right)$ olarak elde edilmektedir. Buradan, önermede belirtilen sonuçlar elde edilebilir.

Önerme 2, $\sigma_{L}<\sigma_{G} \sqrt{\pi}$ şartı sağlandığında Laplace kanalının düşük güç seviyelerinde daha iyi performansa sahip olduğunu, Gauss kanalının ise daha yüksek güç seviyelerinde daha iyi performans gösterdiğini belirtmektedir. Dolayısıyla, sistemin ortalama güç kısıtlamasına bağlı olarak zaman paylaşımı şeklinde her iki kanalı kullanmanın tek kanala göre daha iyi performans gösterebileceğini ifade etmektedir. Kanal anahtarlama oranları ve harcanacak güç seviyeleri denklem (1)'de belirtilen eniyileme probleminin çözümü ile elde edilebilir.

\section{Sayısal Sonuçlar}

Bu bölümde denklem (1)'de verilen eniyileme problemi farklı senaryolar için çalışılmış ve optimal stratejiler belirlenmiştir. İlk olarak, standart sapma değerleri $\sigma_{L}=2$ ve $\sigma_{G}=1$ olan Gauss ve Laplace gürültülü kanallar seçilmiştir. $\mathrm{Bu}$ senaryoda ortalama hata olasılığı her iki kanal için şekil 1'de sunulmuştur.

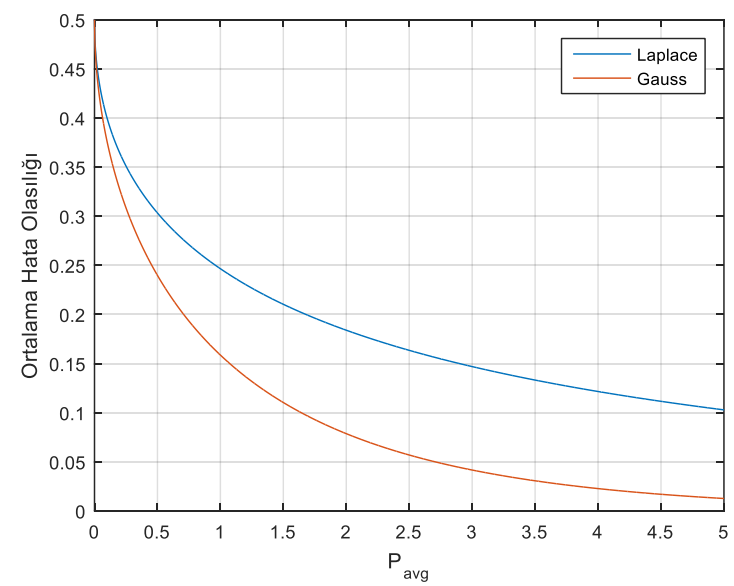


Şekil 1. Gauss ve Laplace gürültülü kanallar için ortalama hata olasılıkları $\left(\sigma_{L}=2\right.$ ve $\left.\sigma_{G}=1\right)$

Grafik incelendiğinde, bütün $P_{a v g}$ değerleri için Gauss gürültülü kanal ile daha düşük ortalama hata olasılığ $\mathrm{Bu}$ durumda, optimal strateji Gauss kanalı üzerinden iletim şeklinde olmalıdır. Önerme 1 'de belirtilen koşul sağlandiğından, bu beklenen bir sonuçtur. Ayrica, Lemma 1'de belirtildiği gibi belirli bir seviyenin üzerindeki ortalama güç değerleri için Gauss kanalı daha düşük ortalama hata olasılığg vermektedir.

Gauss ve Laplace gürültülü kanallar için bir diğer senaryoda, standart sapma değerleri eşit olarak $\left(\sigma_{L}=\sigma_{G}=1\right)$ seçilmiştir. Bu senaryoda, ortalama hata olasılıkları farklı güç kısıtlama değerleri için şekil 2'de gösterilmiştir.

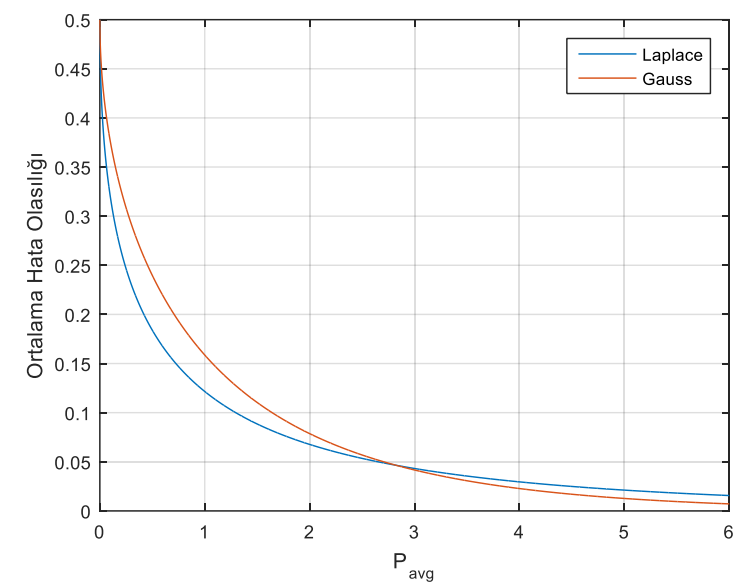

Şekil 2. Gauss ve Laplace gürültülü kanallar için ortalama hata olasilikları $\left(\sigma_{L}=1\right.$ ve $\left.\sigma_{G}=1\right)$

Grafik incelendiğinde, $P_{a v g}=2.8238$ için her iki kanal ile eșit ortalama hata olasılığı elde edildiği (0.0464) gözlemlenmektedir. Ortalama hata olasılığı açısından, daha düşük $P_{a v g}$ değerleri için Laplace gürültülü kanal daha iyi performans verirken, daha yüksek $P_{\text {avg }}$ değerleri için Gauss gürültülü kanal daha iyi performans vermektedir.

Önerme 2'de belirtilen koşul $\left(\sigma_{L}<\sigma_{G} \sqrt{\pi}\right)$ sağlandığından, Şekil 2'de gözlemlenen sonuçlar beklenen sonuçlardır. $P_{a v g}$ değerine bağlı olarak optimal strateji her iki kanalın zaman paylaşımlı olarak kullanımı şeklinde olabilir. Dolayısıyla, denklem (1)'de belirtilen eniyileme probleminin çözümü ile optimal strateji belirlenmektedir.
Denklem (1)'de belirtilen eniyileme probleminin çözümü farklı $P_{a v g}$ değerleri için Tablo 1'de sunulmuştur.

Tablo 1. Farklı $P_{\text {avg }}$ değerleri için denklem (1)'de belirtilen eniyileme probleminin optimal çözümü

\begin{tabular}{|c|c|c|c|}
\hline$P_{\text {avg }}$ & $\alpha$ & $P_{G}$ & $P_{L}$ \\
\hline 2 & 0 & --- & 2 \\
\hline 3 & 0.8127 & 3.1141 & 2.5049 \\
\hline 4 & 1 & 4 & --- \\
\hline
\end{tabular}

Tablo 1 incelendiğinde, $P_{a v g}=2$ için optimal stratejinin Laplace kanalı üzerinden $P_{L}=2$ olacak şekilde iletim olduğu gözlemlenmektedir. İletim için maksimum güç seviyesinin kullanımı $\left(P_{L}=P_{a v g}\right), \quad e_{L}(p)$ tekdüze azalan dişbükey bir fonksiyon olduğundan beklenen bir sonuçtur.

Aynı tabloda, $P_{a v g}=4$ için optimal çözümün Gauss kanalı üzerinden maksimum güç seviyesinde iletim şeklinde olduğu görülmektedir. $e_{G}(p)$ tekdüze azalan dışbükey bir fonksiyon olduğundan maksimum güç seviyesinde iletim $\left(P_{G}=P_{a v g}\right)$, beklenen bir sonuçtur.

Tablodaki ilginç sonuç, $P_{a v g}=3$ durumunda gözlemlenmektedir. $\mathrm{Bu}$ durumda, optimal çözümün 0.8127 oranında Gauss kanalı ve 0.1873 oranında ise Laplace kanalı üzerinden iletim şeklinde olduğu gözlemlenmektedir. Gauss ve Laplace kanalları için harcanan ortalama güç seviyeleri sırasıyla 3.1141 ve 2.5049 olarak elde edilmiştir. $\mathrm{Bu}$ durumda, sistemin harcadığ 1 ortalama gücün $P_{\text {avg }}=3$ seviyesinde olduğu görülmektedir. $\mathrm{Bu}$ strateji için elde edilen ortalama hata olasılığ $\alpha e_{G}\left(P_{G}\right)+(1-\alpha) e_{L}\left(P_{L}\right)=0.0415 \quad$ olarak hesaplanmaktadır.

$P_{a v g}=3$ güç seviyesi için tek kanal üzerinden iletimin nasıl bir performans vereceği merak edilebilir. $\mathrm{Bu}$ durumda, $P_{a v g}>p^{\dagger}$ olduğundan Gauss kanalı Laplace kanalına göre daha iyi performans vermektedir. Gauss kanalı üzerinden, bu güç seviyesinde iletim yapılması 
durumunda ortalama hata olasılığ 0.0416 olarak hesaplanmaktadır. Dolayısıyla, zaman paylaşımlı kanal kullanımının ortalama hata olasılığını iyileștirdiği görülmektedir. Ayrıca, Lemma 1'de belirtilmiş olduğu gibi belirli bir güç seviyesinden sonra Gauss gürültülü kanalının Laplace gürültülü kanala göre daima daha iyi performans sergilediği gözlemlenmektedir.

\section{Sonuçlar}

$\mathrm{Bu}$ çalışmada, verici ve alıcı arasında Gauss gürültülü ve Laplace gürültülü kanallar bulunan ikili iletişim sistemleri çalışılmıştır. Ortalama güç kısıtlaması altında, ortalama hata olasılığını en küçülten optimal stratejiler belirlenmiștir. Öncelikle, Gauss gürültülü kanal üzerinden iletimin optimal olduğu, zaman paylaşımlı kanal kullanımının önerilmediği koşul belirlenmiştir. Daha, sonra zaman paylaşımlı kanal kullanımının tek kanallı iletime göre performansı iyileştirebileceği koşul belirlenmiştir. Kuramsal sonuçları doğrulamak maksadıyla sayısal örnekler sunulmuştur.

\section{Kaynaklar}

[1] C,. Goken, S. Gezici, O. Arıkan, "Optimal stochastic signaling for power-constrained binary communications systems", IEEE Transactions on Wireless Communications, vol. 9, no. 12, pp. 36503661, Dec. 2010.

[2] C,. Goken, S. Gezici, O. Arıkan, "Stochastic signaling in the presence of channel state information uncertainty", Digital Signal Processing, vol. 23, no. 2, pp. 635-645, Mar. 2013.

[3] H. Chen, P. K. Varshney, S. M. Kay, and J. H. Michels, "Theory of the stochastic resonance effect in signal detection: Part I-Fixed detectors," IEEE Trans. Sig. Processing, vol. 55, pp. 3172-3184, July 2007.

[4] H. Chen, P. K. Varshney, S. M. Kay, and J. H. Michels, "Theory of the stochastic resonance effect in signal detection: Part II-Variable detectors," IEEE Trans. Sig. Processing, vol. 56, no. 10, pp. 5031-5041, Oct. 2007.

[5] S. Loyka, V. Kostina, and F. Gagnon, "Error rates of the maximumlikelihood detector for arbitrary constellations: Convex/concave behavior and applications," IEEE Trans. Information Theory, vol. 56, no. 4, pp. 1948-1960, April 2010.
[6] B. Dulek and S. Gezici, "Detector randomization and stochastic signaling for minimum probability of error receivers," IEEE Trans. Commun., vol. 60, no. 4, pp. 923-928, Apr. 2012

[7] M. E. Tutay, S. Gezici, and O. Arikan, "Optimal detector randomization for multiuser communications systems," IEEE Trans. Commun., vol. 61, pp. 2876-2889, July 2013.

[8] M. Azizoglu, "Convexity properties in binary detection problems," IEEE Trans. Info. Theory, vol. 42, no. 4, pp. 1316-1321, July 1996

[9] B. Dulek, M. E. Tutay, S. Gezici, and P. K. Varshney, "Optimal signaling and detector design for M-ary communication systems in the presence of multiple additive noise channels," Digital Signal Processing, vol. 26, pp. 153-168, Mar. 2014.

[10] M. E. Tutay, S. Gezici, H. Soganci, and O. Arikan, "Optimal channel switching over Gaussian channels under average power and cost constraints," IEEE Trans. Commun., vol. 63, pp. 1907-1922, May 2015 .

[11] S. Verdu, Multiuser Detection. 1st ed. Cambridge, UK: Cambridge University Press, 1998.

[12] H. V. Poor, An Introduction to Signal Detection and Estimation. New York: Springer-Verlag, 1994.

[13] A. Leon-Garcia, Probability and Random Processes for Electrical Engineering. 2nd ed. Addison, Wesley, 1994.

[14] J. G. Proakis and M. Salehi, Communication Systems Engineering, NJ, Englewood Cliffs:Prentice-Hall, 1994.

[15] G. B. Thomas, M. D. Weir, J. Hass, and F. R. Giordano, Thomas' Calculus. Addison Wesley, 11 ed., 2004. 